\title{
A review of family carers' experiences of hospital discharge for people with dementia, and the rationale for involving service users in health research
}

\author{
This article was published in the following Dove Press journal: \\ Journal of Healthcare Leadership \\ 22 June 2015 \\ Number of times this article has been viewed
}

\section{Carole Mockford \\ Division of Health Sciences, Royal College of Nursing Research Institute, University of Warwick, Coventry, UK}

\begin{abstract}
In the UK, service user involvement is an important factor in health policy, and obtaining research funding. It may be helpful in expanding our knowledge in areas where research evidence is poor such as experiences of hospital discharge planning for the family carers of people with dementia.

Methods: A rapid review. All study designs published in the English language were eligible for inclusion. Databases included: Medline, Embase, CINAHL, PsycInfo, Cochrane library and Web of Knowledge. A qualitative analysis was undertaken.

Results: Four themes were identified: preparation for hospital discharge - dissatisfaction with being kept informed, discharge arrangements and management of conditions; little time to prepare. Communication between staff and families at discharge - insufficient communication regarding services, not being listened to and being undervalued as a resource could compromise post-discharge care. Support services post discharge - carers need help negotiating, and working with, services with regard to timing, and meeting requirements. Coping post hospital discharge - inadequate understanding about ability to cope, and patient's impairment, and family conflict over care may lead to unnecessary re-admission to hospital, or long term care. Evidence of specialist dementia models at discharge is described.

Discussion: Carers are not always involved in hospital discharge planning as well as they might be. Issues are complex and depend on a number of factors. Poor communication can be overcome and carers can be better supported to cope post discharge as illustrated in the dementia models. The evidence base is weak but the absence of evidence does not indicate an absence of good practice.
\end{abstract}

Conclusion: Carers who may feel their world is far removed from the academic world may not ordinarily participate in research studies. Service users, as co-researchers, may be able to improve trust and rapport between research and communities, collect fresh insights and gain deeper and more insightful data from participants.

Keyword: hospital discharge, carers, dementia, user involvement

\section{Introduction}

In the UK, service user involvement has become an important factor in health policy and in obtaining research funding. Both are important drivers in user involvement and patient engagement in research. The National Institute of Health Research, ${ }^{1}$ funded through the Department of Health to improve the health and wealth of the nation through research, is a major funder. A key requirement of their funding application is for applicants to explain how user involvement has helped to develop the research
Correspondence: Carole Mockford Division of Health Sciences, Royal College of Nursing Research Institute, University of Warwick, Coventry CV4 7AL, UK

Tel +4402476150627

Email carole.mockford@warwick.ac.uk 
proposal and how service users will be included in the project. The success of this initiative, documented in a recent report, is anticipated to shape the next 10 years of public involvement in research. ${ }^{2}$ The National Institute of Health Research also funds INVOLVE, ${ }^{3}$ an organization that supports active patient and public involvement in research in the National Health Service (NHS) and public and social care. The Alzheimer's Society ${ }^{4}$ also has a large network of actively involved service users. Priorities for research are regularly set by the Quality Research in Dementia lay review panel and committees in Alzheimer's Society's governance framework. Lay members also review, and comment on, the funding applications. With this in mind, research funding applications in health research are often at a disadvantage where service users are not involved at some stage of the project development and/or research process.

A systematic review ${ }^{5}$ found clear evidence that user involvement can have a positive effect on the development of research studies. It found that by giving a voice to service users at an early stage, proposed topics, aims, and methods could be challenged for importance and relevance to those with the condition under investigation. Service users enable easier access to hard-to-reach groups, ${ }^{6}$ and as coresearchers who may have similar experiences, they can identify issues that may seem insignificant to an academic audience, can make sense of results from their own perspectives creating meaningful interpretations, ${ }^{7}$ and are more likely to act as advocates for the findings, ${ }^{5}$ thereby widening dissemination to places where researchers, with limited time on a study, may not normally have time to go to beyond presenting to academic audiences, writing academic papers, and submitting reports to funders. Another systematic review ${ }^{8}$ found that user involvement in health and social care services has been shown to be beneficial in cancer and stroke services and health service planning, where there was some evidence that services were reorganized, relocated, or enhanced and additional services were put in place as a result. Often the changes were simple but made a major difference to those using the services.

This paper seeks to understand the scope of involving service users as lay researchers in health research, from a researcher's perspective, where solutions might be found at the ground level, ie, from the bottom up, rather than from reports and policy initiatives where the agenda is already likely to be set by policymakers and professionals, ie, from the top down. The topic under investigation is hospital discharge planning. This paper reports on the global scale of dementia, and the consequences for people with dementia and their families experiencing poor decision-making at hospital discharge. It presents a literature review focusing on hospital discharge planning from the perspective of family carers of people with dementia. It then argues a case for the involvement of service users as lay researchers who may be able to further our knowledge of carers' experiences that academic researchers may miss.

In recent years, the Department of Health in the UK has been a driver for improving care and support for people with dementia and dementia research;, ${ }^{9} 10$ the latter previously lagged behind other conditions such as cancer research, as by comparison, dementia research has received far less government funding. Following the G8 summit on dementia in $2013,,^{11}$ the government has greatly increased funding into both dementia care and support and research. ${ }^{12}$ The Prime Minister's Challenge on Dementia $2020^{13}$ focuses on making England the best country in the world for dementia care and support and in undertaking research into dementia and other neurodegenerative diseases. Two factors emerged from the G8 dementia summit ${ }^{11}$ and the latest response from the government: ${ }^{13}$ a call for "greater innovation to improve the quality of life for people with dementia and their carers while reducing emotional and financial burden" 11 and the aspiration that dementia research takes place through a partnership of patients, researchers, funders, and society. ${ }^{13}$

This paper addresses:

- the scope of the problem on leaving the hospital with dementia;

- the experience of hospital discharge planning for family carers of people with dementia through a rapid review of the literature; and

- where service user involvement might be meaningful in the development of the evidence base.

\section{The scope of the problem on leaving the hospital with dementia}

Dementia is a fast growing worldwide problem. An estimated 35.6 million people lived with dementia in 2010, and this number is projected to double every 20 years, so that by 2050 , the figure could be as high as 115.4 million. The global annual cost of dementia is reported to be $\$ 604$ billion. ${ }^{14}$ The G8 summit on dementia in 2013 identified dementia as a major disease burden having an increasingly significant financial impact on the world's health economy. ${ }^{11}$

In the UK, 835,000 people are currently estimated to have dementia, costing the economy $£ 26$ billion annually. ${ }^{15} \mathrm{Up}$ to $70 \%$ of acute hospital beds are occupied by older people, many with chronic illnesses, and it is estimated that almost half of these patients have some form of cognitive impairment, including dementia. ${ }^{9}$ A person with dementia may need complex care following hospital discharge, particularly as he/she may have varied levels of awareness, as well as multimorbidities, and increased dependency on a family member. ${ }^{16}$ 
The importance of hospital discharge planning cannot be underestimated as it plays a vital role in bridging the gap between acute care and community support. Research has confirmed a direct link between the quality of discharge planning for people with dementia and readmission to hospital. ${ }^{17,18} \mathrm{Leg}$ islation in the UK aims to ensure that patients are not kept in hospital unnecessarily ${ }^{19}$ and gives responsibility to health care professionals to provide a smooth discharge from hospital, to ensure that community services are in place and that the patient and their carer are supported once they are home. ${ }^{20,21}$

Taking the time to get hospital discharge planning right can delay discharge and block urgently needed hospital beds, ${ }^{22}$ but getting it wrong can place extra (or new) burden on family carers to cover the gap until services can be operationalized (if at all) or lead to premature, and often permanent, admission to a care home. ${ }^{23}$

People with dementia who are admitted to acute hospitals have a higher rate of mortality, increased morbidity, and higher risk of entering institutional care ${ }^{24}$ than people without dementia. Hastily made decisions at hospital discharge can result in poor health outcomes ${ }^{25}$ and life-changing decisionmaking. A large percentage of people with dementia currently live in care homes (30\%), and two-thirds of residents in care homes have dementia. One-third of patients with dementia are discharged to a care home from hospital., ${ }^{926}$ Neither the cost of this nor the planned workforce needed to cope with this has been planned for. ${ }^{9}$ Behavioral and psychological symptoms of dementia, which include agitation, aggression, calling out, sleep disturbance, wandering, and apathy, are the key factors that lead to carer strain and are reasons for institutionalization when families can no longer cope. ${ }^{27}$ Families generally want to stay together as long as possible, and they find the decisionmaking around care homes difficult and fraught with feelings of guilt and failure. ${ }^{28}$ Premature admission to a care home is not only expensive for the health economy but can also be devastating for the person with dementia and their families.

\section{Recent enquiries and reports}

It has been recognized that many hospital staff have a poor knowledge of dementia. This has led to investigations into the experiences of people with dementia in hospital and at hospital discharge. Hospital discharge processes and those who implement them are governed by the Mental Capacity Act (2005) ${ }^{29}$ and common law in England and Wales. There are concerns that there is poor compliance with the Act when people with dementia leave hospital, particularly as many patients with dementia who are admitted to hospital from their homes are discharged to a long-term care home. ${ }^{30}$ An ethnographic study ${ }^{31}$ found that family carers were often ill-equipped to challenge professional, best interest, decisions to place a person with dementia in long-term care from the hospital, although there is an implicit assumption within the Mental Capacity Act (2005) ${ }^{29}$ that they are able to safeguard the patient's interest and rights where appropriate. However, the Act is reported to have "suffered from a lack of awareness and a lack of understanding" (p 7) by professionals, individuals, families, and carers, and its implementation is lagging behind the legislation. ${ }^{30}$

Questions have been raised about the extent to which risks and resources take the lead in decision-making and whether these decision makers adequately consider improved support at home and the roles of relatives..$^{30}$

Extensive consultation with professionals, carers, and people with dementia has led to several national reports, guidelines, standards, and recommendations issued by the Department of Health, health organizations, and charitable bodies, which have acknowledged the lack of appropriate health and social provision given to people with dementia and their carers. ${ }^{9,23,26,32-35}$ There is little research evidence to inform key decision makers and policy makers, but emerging insights from recent commissioned reports point to some major gaps in the process that can only negatively impact on patients and their families. ${ }^{23,32,34,35}$ Key issues have been raised, which included the following:

1. The identification of poor quality, and possibly unsafe, hospital discharges, which include a rush to discharge: without a proper assessment, without proper liaison with social services, and without a proper package of care arranged. ${ }^{35,36}$

2. Families reporting specific concerns with the timing of hospital discharges ${ }^{32}$ with some hospital records showing that no notice, or less than 24 hours notice, of discharge had been given. ${ }^{34}$

3. A lack of coordination between hospitals and care providers at discharge leading to delays in care packages, eg, home care and intermediate care. ${ }^{23}$

4. Few hospitals having guidelines that include asking the main carer about their wishes and ability to provide care and support after hospital discharge ${ }^{34}$ and a lack of coordinated services for carers which takes into account their needs..$^{37}$ Despite carrying the responsibility of caring, a report ${ }^{34}$ found that only $40 \%$ of hospitals had a process for including families at discharge and that just half of the hospitals had guidelines for involving families in arrangements for discharge and support services.

The Carers Strategy ${ }^{37}$ is a 10-year strategy that focuses on the provision of personalized services that are fit for the purpose of carers and that aim to support people and their carers in their own homes to the maximum degree possible. By keeping 
people in their homes, family carers save the UK NHS economy around $£ 11$ billion per year in dementia care, more than cancer and heart disease together. ${ }^{38}$ The financial cost of dementia to the economy totals $£ 26.3$ billion, and two-thirds of this is paid for by the people with dementia and their families by way of care and private social care. Unlike other diseases such as heart disease and cancer where NHS care is free, with dementia, although classed as a physical disease of the brain, the majority of care provided is means-tested social and personal care, which falls under social care services rather than health care services. ${ }^{39}$ Families, often struggling to cope with their caring role, may find social care services have to be paid for but which are reported to be financially at breaking point. ${ }^{40}$

Fiscal restraints have been affecting the health and social care services in recent years, ${ }^{41,42}$ and the social care budget is reported to have fallen by $20 \%,{ }^{43}$ cutting some services and forcing others to continually reevaluate their working practices. Specialist services for people with dementia who are discharged from hospital are rare. ${ }^{23}$

With the older population rising and with dementia numbers increasing at an alarming rate, it is clear that more and more family carers will be bearing the brunt of the care burden.

Currently, there is little evidence documenting carer and patient perspectives of health and social care services during and after hospital discharge, 22 little published evidence to guide best practice for people with dementia who leave hospital and return home, ${ }^{25}$ little evidence that discharge planning meets best practice, ${ }^{18}$ and there is an absence of evidence on good outcomes for carers of people with dementia at hospital discharge. ${ }^{32}$

There is a small window of opportunity in the period from hospitalization to hospital discharge where people with dementia and their families and carers can find their lives changed forever. This is a timely opportunity to assess the research evidence base on the experiences of health and social care services received from the point of hospital discharge to home from the perspective of carers of people diagnosed with dementia, as they offer valuable insight into what the person they care for is experiencing and communicating. ${ }^{44}$ This could inform health and social care services and enable the person with dementia and their families to have a better quality of life after a hospital episode and possibly delay admission to long-term care.

\section{The experience of hospital discharge planning for family carers of people with dementia: a literature review}

A literature review was conducted that examined the evidence base for family carers' perspectives of hospital discharge for people with dementia, and it found a paucity of reported studies across the globe.

\section{Data sources}

A multifield search was made using Ovid Medline (1946week 1 of February 2015) Embase (1980-2015), CINAHL (1991-February 2015), PsycINFO (1800-February 2015), Cochrane Library (till February 2015), and Web of Knowledge (1898-February 2015) for all published papers written in the English language. Search terms included caregiver, carer, dementia, hospital, and discharge. Inclusion criteria were any study design, informal or family carers, a person with dementia, and at hospital discharge. Exclusion criteria were conference proceedings, think pieces, and editorials.

\section{Study selection}

The literature search produced 139 hits. From these studies, a citation and key author search was also conducted. Most studies were discarded as they referred to diagnosis and treatment, hospital to other care facilities, patient health, did not include carers, were not dementia studies or the data were provided by the carer on behalf of the patient. Ten studies (13 papers) were included in this review.

Of the ten studies, four (five papers) were from the USA, ${ }^{17,25,45-47}$ three from the UK, ${ }^{48-50}$ two (four papers) from Australia, ${ }^{51-54}$ and one from Taiwan. ${ }^{55}$ Three studies used a cross-sectional telephone survey, ${ }^{17,45,47}$ three studies conducted qualitative interviews with carers, ${ }^{49,51-53,55}$ and three studies utilized a mixed methods approach. ${ }^{25,48,54}$ One study performed a secondary statistical analysis from a parent study. ${ }^{50}$

A narrative synthesis was performed as the diversity of data collection processes made it impossible to collate the data in any numerical form, such as a meta-analysis. It involved familiarization with the papers and then identification of emergent themes. The assessment of the quality of the studies was guided by the Centre for Reviews and Dissemination. ${ }^{56}$ A formal assessment of quality was not conducted as the scope and purpose of the studies was diverse and quality varied, but no study was excluded on quality thresholds.

\section{Results}

Focusing on the family carer of a person with dementia, the findings presented themselves in four themes: preparation for hospital discharge, communication between staff and families at discharge, support services post discharge, and coping post-hospital discharge. Lastly, there is some evidence of specialist dementia models being introduced into hospitals to support families after hospital discharge, and this is also reported. 


\section{Preparation for hospital discharge}

The importance of discharge planning was rated more highly by family carers than the patients who expected that their family would deal with this when the time came. ${ }^{54}$ Carers reported being dissatisfied with being kept informed, discharge arrangements, and the management of medical problems, even though the hospital in one study considered person-centered care to be a standard practice and deliberate attempts had been made to improve carer and patient experience.$^{50}$ When asked, carers described the exhaustion and despair they experienced and the challenges they faced at hospital discharge, ${ }^{47}$ such as seeking out information about the patient's condition, post-discharge care, and support services..$^{51}$ There was concern that there was little time to prepare for the discharge ${ }^{53}$ and an immediate need for support services after discharge. ${ }^{55}$ It was reported in one Australian study that carers felt that discharge planning was ad hoc with an absence of any organization. ${ }^{51,52}$ There was significant association with levels of carer strain and their psychological well-being measured at hospital admission, ${ }^{50}$ and carers reported that they needed long-term and continuous emotional support to cope post-hospitalization. ${ }^{55}$ The lack of available and affordable resources or possible complications presented by family carers are not always considered in discharge planning. ${ }^{17}$ The complex nature of dementia and the unrealistic expectations of some families often make disease management difficult. ${ }^{47}$

\section{Communication between staff and families at discharge}

There was insufficient communication with services about the patient's needs, and carers felt that the staff did not listen to them at the hospital and undervalued them as a resource..$^{51,53}$ In this Australian study, ${ }^{51,53}$ carers were mostly ignored in discharge planning, leaving them unprepared and unable to ask questions about the patient's care and thus compromising post-discharge care. Carers felt that they were given little time to absorb new information from hospital staff in order to make critical decisions, ${ }^{17}$ and they sometimes needed direction ${ }^{47}$ and help in searching for relevant resources, ${ }^{17,25,47}$ especially when the patient was in hospital. Informing families about the availability of support or support services varied, where some professionals were more likely to give helpful advice to families than others. ${ }^{49}$

\section{Support services post discharge}

A family carer played a key role in the transition back home, and an early study by Cox and Verdieck ${ }^{45}$ found that if there were formal services in place and the carer had assistance with medication, bathing and supervision, and support and counseling, then the patient tended to return home from hospital rather than making the transition to a care home. However, carers needed to know how to negotiate service provision and manage the care of the patient in conjunction with services, ${ }^{25}$ particularly with regard to timing and levels of service provision that may fail to meet the families' requirements. ${ }^{51,53}$ Sometimes families were let down when the services failed to turn up due to a breakdown in communication. ${ }^{51}$

\section{Coping post discharge}

The immediate difficulties faced by carers were attributed to the carer being unrealistic about their ability to care and not accepting the severity of the patient's functional impairment. ${ }^{17}$ Carers did not always continue with hospital discharge instructions or individual care plans once the patient was home, which may stem from a lack of understanding of both post-hospital care and the patient's condition. ${ }^{46}$ Carers also found it difficult to coordinate appointments with transport, to manage specialist care, or occasionally refused the help offered ${ }^{46}$ In one study, ${ }^{17} 18 \%$ of patients with dementia were readmitted to the hospital within 1 month, with much of the predictive factors being attributed to family carers' inability to carry on, eg, the patient needed heavy care, the patient had problematic behavior, or the carer lacked social support. Long-term family conflict over care provision may lead to the person with dementia being admitted into a care home. ${ }^{47} \mathrm{In}$ measuring levels of stress in carers of people with dementia, Gage et $\mathrm{al}^{48}$ found that this was significantly reduced when the person they cared for was admitted to residential care.

\section{Dementia-specialist care models}

There are some dementia-specialist care models in place, but the research evidence for these is patchy. Two studies based on care models and conducted in the USA ${ }^{25,47}$ offer education, information, behavioral management, long-term care planning, support for the patients with dementia and their carers, and training of carers. One of these ${ }^{25}$ reported that the specialized staff leading a tailored care program were in a position to authorize delayed discharge until appropriate services were in place at home. Each care package was customized to the individual patient and carers' needs. ${ }^{25,47}$ The patient and carer were supported post discharge, eg, carers were guided by nominated health professionals offering information; identifying problem areas, symptom management, and interventions; and providing linkages for long-term planning. One program was goal oriented and time limited 
to 6 weeks during the period of transition from hospital to home, ${ }^{25}$ whereas the other was ongoing from diagnosis and included time in hospital. ${ }^{47}$

\section{Discussion of the literature review}

Comparing the findings from this review with the UK reports on hospital discharge of people with dementia, it is clear that carers have not been involved in the discharge process as well as they might have been. This is not to say that there is no good practice across hospitals, but the good practice is not always reported. The literature review found four themes of importance to carers: preparation for hospital discharge, communication between staff and families at discharge, support services after discharge, and coping post discharge. Internationally, hospital discharge policies may differ between countries, but there were some commonalities across the literature.

Official reports ${ }^{35,36}$ identified a discharge that may be rushed, unplanned, and without proper liaison with social services. The research literature found that carers had little time to prepare for a discharge ${ }^{53}$ or absorb new information in order to make critical decisions. ${ }^{17}$ The experience may have been better if someone had spent some time with the family, particularly at this crucial time, when a hasty decision can lead to long-term institutional care.

It has been reported that a few hospitals have guidelines for including carers in hospital discharge planning ${ }^{34}$ and there is a lack of coordinated services to consider the carers' needs. ${ }^{37}$ The literature found that hospital discharge planning is very important to many carers ${ }^{54}$ and that carers are seen as playing a key role in the patients' discharge. ${ }^{45}$ However, carers reported a lack of communication with health professionals, not being listened to and feeling undervalued as a resource. ${ }^{53}$ A lack of communication and professional support may explain the reasons why some carers may not comply with hospital instructions, care plans, or appointments or refuse any help offered. ${ }^{46}$ Hospital discharge is an ideal time to speak to the patient and family together, to plan for the immediate future, and to possibly avoid readmission to the hospital.

The psychological well-being of carers of people with dementia is often reported as poor, and hospital discharge can be a time of significant change for both the patient and carer and many families need emotional and practical support. ${ }^{57}$ Some specialist services are being developed and have shown some promising results but are a few in number and differ in care models. ${ }^{25,47}$

Key messages from this literature review indicate that little evidence is available: a) for firm conclusions to be reached about how carers experience hospital discharge when the patient has dementia; b) to understand why carers report dissatisfaction with hospital staff and particularly a lack of communication at discharge even when the hospital might think they are being person centered; c) to know why services appear to fail families once the patient is back in the community; and d) to describe why there is discordance between hospital care plans and carer behavior post discharge. There may be something to learn from the dementia-specialist care models described in the two US studies, ${ }^{25,47}$ however, much more research is needed in this complex area. The findings from this review are limited as there were a few studies that included the combination of terms: carers, people with dementia, and hospital discharge. Other terms such as frail elderly or cognitive impairment might reveal more studies. Despite the lack of research evidence, the number of reports compiled on people with dementia as inpatients in acute hospitals and on leaving the hospital, however, illustrates the value of examining this topic in more detail. If more communication and support for carers are all that are needed, then simple, cost-effective changes may make a major change to families' experiences and enable more time at home for people with dementia.

\section{Where service user involvement might be meaningful in the development of the evidence base}

There are many forces that are shaping service delivery in health care, not only policies and budgets but also the needs and wishes of patients and their families. An Australian study ${ }^{51}$ recommended that a comprehensive discharge plan should be in place that includes the carer as a partner in care and offers a person-centered approach to the patients' unique circumstances. Oyebode ${ }^{58}$ also championed the idea of carers as partners in mental health services where the partnership can be described as on a continuum from a point where the focus of services lies solely with the patient's needs to a shared partnership consisting of patient, carer, and services working toward shared goals. However, families and those with dementia may not have the time or energy to make their argument heard, although many will participate in research studies in the hope that it will make a difference to others in the same situation. Involving service users in health research has already been shown to gain fresh insights, and it was reported that, as lay coresearchers, deeper and more insightful data were collected, rapport was improved with participants, and trust was gained between research and communities, all of 
which have helped with the success of research studies. ${ }^{59}$ Service users as coresearchers may reach those who would not ordinarily participate in empirical studies and may be helpful in expanding our knowledge in areas where research evidence is poor, such as where data are difficult to obtain, eg, where patients and carers are too involved in trying to cope with post-hospitalization recovery and negotiating social care.

\section{Conclusion}

Family carers are vital to the ongoing recovery of the patient who has dementia and in the prevention of unnecessary readmission to hospital or premature admission to long-term care. The literature review has highlighted several problem areas that lack research evidence. Gathering evidence from those directly involved, particularly those experiencing hospital discharge and care services, is key to developing services that are not only personalized but also give control to families to organize their lives as they want to. However, important, information may not be divulged by families to researchers, whose academic world seems miles apart from theirs, or they refuse to participate, and we are missing this evidence that may contribute to improving services. Service users who have similar experiences may be able to access hard to reach participants, may be able to ask different but more pertinent questions, and offer personal insights that may be missed by professionals or academic researchers when developing a research study, collecting data, analyzing data, and disseminating research findings. Service user involvement in health research has the potential to contribute a unique understanding to the development of the evidence base, which is relevant to those receiving services and is relevant to those providing the services.

\section{Acknowledgments}

Many thanks to the colleagues at the Royal College of Nursing Research Institute for their support and comments on this paper, particularly Professor Kate Seers and Dr Elizabeth Tutton. Thanks also to the reviewers of this paper for their helpful advice and comments, which have been incorporated into this paper.

\section{Disclosure}

The author reports no conflicts of interest in this work.

\section{References}

1. National Institute of Health Research. Available from: http://www. nihr.ac.uk/funding/public-involvement-in-your-research.htm. Accessed March 16, 2015.
2. National Institute of Health Research. Going the Extra Mile: Improving the Nation's Health and Well-being Through Public Involvement in Research; 2015. Available from: http://www.nihr.ac.uk/newsroom/ get-involved-news/going-the-extra-mile-a-strategic-review-of-publicinvolvement-in-the-national-institute-for-health-research/2739 2015. Accessed April 10, 2015.

3. INVOLVE. Available from: http://www.invo.org.uk/. Accessed March 16, 2015.

4. Alzheimer's Society. Available from: http://www.alzheimers.org.uk/ site/scripts/documents.php?categoryID=200426. Accessed March 16, 2015.

5. Brett J, Staniszewska S, Mockford C, et al. Mapping the impact of patient and public involvement on health and social care research: a systematic review. Health Expect. 2014;17(5):637-650.

6. Patterson S, Trite J, Weaver T. Activity and views of service users involved in mental health research: UK survey. $B r J$ Psychiatry. 2014;205:68-75.

7. Ellins J, Glasby J, Tanner D, et al. Understanding and Improving Transitions of Older People: A User and Carer Centred Approach. Final Report. NIHR Service Delivery and Organisation Programme. SDO: London; 2012.

8. Mockford C, Stanisewska S, Griffiths F, Herron-Marx S. The impact of patient and public involvement on UK NHS health care: a systematic review. Int J Qual Health Care. 2012;24(1):28-38.

9. Department of Health. Living Well with Dementia: A National Dementia Strategy. DH: London; 2009.

10. Department of Health. Dementia Challenge. London: DH; 2012.

11. G8UK. G8 Dementia Summit Declaration. London: DH; 2013.

12. Department of Health. The Care Act 2014. London: The Stationery Office; 2015.

13. Department of Health. Prime Minister's Challenge on Dementia 2020. London: DH; 2015.

14. World Health Organisation. Dementia - A Public Health Priority. Switzerland: WHO press; 2012.

15. Alzheimer's Society. Opportunity for Change. London: Alzheimer's Society; 2014.

16. Carers Trust. Road Less Rocky - Supporting Carers of People with Dementia. London: Carers Trust; 2013.

17. Cummings S. Adequacy of discharge plans and rehospitalisation among hospitalised dementia patients. Health Soc Work. 1999;24(4): 249-259.

18. Bauer M, Fitzgerald L, Haesler E, Manfrin M. Hospital discharge planning for frail older people and their family. Are we delivering best practice? A review of the evidence. J Clin Nurs. 2009;18(18):2539-2546.

19. Department of Health. Discharge from Hospital - Getting It Right for People with Dementia. London: DH; 2003.

20. Department of Health. Carers (Equal Opportunities) Act. London: The Stationery Office; 2004.

21. Department of Health. National Framework for Long Term Conditions. London: DH; 2005.

22. Glasby J, Littlechild R, Pryce K. Show Me the Way to Go Home: Delayed Hospital Discharges and Older People - Final Report. Birmingham: Health Services Management Centre, and Institute of Applied Social Studies, University of Birmingham; 2004.

23. Department of Health. Recognised, Valued and Supported: Next Steps for the Carers Strategy. London: DH; 2010.

24. Leung D. Dementia patients and hospital discharge: mind the gap. Dementia. 2011;1(10):1-2.

25. Naylor M, Hirschman K, Bowles K, Bixby B, Konick-McMahon J, Stephens C. Care co-ordination for cognitively impaired older adults and their caregivers. Home Health Care Serv Q. 2007;26(4):57-78.

26. Alzheimer's Society. Counting the Cost and Counting the Cost Summary. London: Alzheimer's Society; 2009.

27. Alzheimer's Disease International. World Alzheimer's Report. London: Alzheimer's Disease International; 2009.

28. Livingston G, Leavey G, Manela M, et al. Making decisions for people with dementia who lack capacity: qualitative study of family carers in UK. BMJ. 2010;341:c4184. 
29. Department of Constitutional Affairs. Mental Capacity Act 2005: Code of Practice. London: The Stationery Office Ltd; 2007.

30. House of Lords. Report of the Select Committee on the Mental Capacity Act 2005; 2014. Accessed April 10, 2015.

31. Emmett C, Poole M, Bond J, Hughes J. A relative safeguard? The informal roles that families and carers play when patients with dementia are discharged from hospital into care in England and Wales. Int J Law Pol Fam. 2014;28:302-320.

32. Association of Directors of Adult Social Services. Carers as Partners in Hospital Discharge. London: Association of Directors of Adult Social Services; 2010.

33. National Institute Clinical Excellence. Dementia Quality Standard. London: National Institute Clinical Excellence; 2010.

34. Young J, Hood C, Gandesha A, Souza R. National Audit for Dementia Care in General Hospitals 2012-13: Second Round Audit Report and Update. London: HQIP; 2011.

35. Royal College of Nursing. Dignity in Dementia: Transforming General Hospital Care. London: Royal College of Nursing; 2011.

36. Bernard C. 'No Help Here'. London: Council and Care, for older people, their families and carers; 2011.

37. Department of Health. Carers at the Heart of 21st Century Families and Communities. London: DH; 2008.

38. Health Foundation. Spotlight on Dementia Care: A Health Foundation Improvement Report. London: Health Foundation; 2011.

39. Alzheimer's Society. Financial Cost of Dementia; 2015. Available from: http://www.alzheimers.org.uk/site/scripts/documents_info. php?documentID=418. Accessed March 16, 2015.

40. Alzheimer's Society. Social Care System in Rapid Decline, Warns Age $U K ; 2015$. Available from: http://www.alzheimers.org.uk/site/scripts/ news_article.php?newsID=2296. Accessed March 16, 2015.

41. Mental Health Foundation. The Mental Health Strategy, System Reforms and Spending Pressures: What Do We Know So Far?; 2015. Available from: http://www.mentalhealth.org.uk/publications/mental-healthstrategy/. Accessed March 16, 2015.

42. Fernandez J-L, Snell T, Wistow G. Changes in the Patterns of Social Care Provision in England: 2005/6 to 2012/13. Canterbury: Personal Social Services Research Unit; 2013.

43. Triggle N. Care Spending 'Cut by Fifth in 10 Years'; 2015. Available from: http://www.bbc.co.uk/news/health-31015807. Accessed March 16, 2015.

44. Chung P, Ellis-Hill C, Coleman P. Carers' perspectives on the activity patterns of people with dementia. Dementia. 2008;7(3):359-381.

45. Cox C, Verdieck M. Factors affecting the outcomes of hospitalized dementia patients: from home to hospital to discharge. Gerontologist. 1994;34(4):497-504.
46. Bradway C, Trotta R, Bixby MB, et al. A qualitative analysis of an advanced practice nurse-directed transitional care model intervention. Gerontologist. 2012;52(3):394-407.

47. Ghatak R. A unique support model for dementia patients and their families in a tertiary hospital setting: description and preliminary data. Clin Gerontol. 2011;34(2):106-172.

48. Gage H, Cheynel J, Williams P, et al. Service utilisation and family support of people with dementia: a cohort study in England. Int J Geriatr Psychiatry. 2015;30(2):166-177.

49. Newans A, Forster D, Kay W. Referral patterns and diagnosis in presenile Alzheimers disease: implications for general practice. Br J Gen Pract. 1994;44:405-407.

50. Whittamore K, Goldberg S, Bradshaw L, Harwood R. Factors associated with family caregiver dissatisfaction with acute hospital care of older cognitively impaired relatives. JAm Geriatr Soc. 2014;62: 2252-2260.

51. Bauer M, Fitzgerald L, Koch S. Hospital discharge as experienced by family carers of people with dementia: a case for quality improvement. J Healthc Qual. 2011;33(6):9-16.

52. Bauer M, Fitzgerald L, Koch S, King S. How family carers view hospital discharge planning for the older person with a dementia. Dementia. 2011;10(3):317-323.

53. Fitzgerald L, Bauer M, Koch S, King S. Hospital discharge: recommendations for performance improvement for family carers of people with dementia. Aust Health Rev. 2011;35:364-370.

54. Hancock K, Chang E, Chenoweth L, Clarke M, Carroll A, Jeon YH. Nursing needs of acutely ill older people. $J$ Adv Nursing. 2003;44(5): 507-516.

55. Shyu Y. The needs of family caregivers of frail elders during the transition from hospital to home: a Taiwanese sample. $J$ Adv Nurs. 2000;32(3):619-625.

56. Centre for Reviews and Dissemination. Systematic Reviews: Guidance for Undertaking Reviews in Health Care. York: CRD; 2009.

57. Plank A, Mazzoni V, Cavada L. Becoming a caregiver: new family carers' experience during the transition from hospital to home. J Clin Nurs. 2012;21:2072-2082.

58. Oyebode J. Carers as partners in mental health services for older people. Adv Psychiatr Treat. 2005;11:297-304.

59. Brett J, Staniszewska S, Mockford C, et al. A systematic review of the impact of patient and public involvement on service users, researchers and communities. Patient. 2014;7(4):387-395.
Journal of Healthcare Leadership

\section{Publish your work in this journal}

The Journal of Healthcare Leadership is an international, peer-reviewed, open access journal focusing on leadership for the health profession. The journal is committed to the rapid publication of research focusing on but not limited to: Healthcare policy and law; Theoretical and practical aspects healthcare delivery; Interactions between healthcare and society and evidence-based practices;
Dovepress

Interdisciplinary decision-making; Philosophical and ethical issues; Hazard management; Research and opinion for health leadership; Leadership assessment. The manuscript management system is completely online and includes a very quick and fair peer-review system. Visit http://www.dovepress.com/ testimonials.php to read real quotes from published authors. 